\title{
The Association Between Anterior Cruciate Ligament Length and Femoral Epicondylar Width Measured on Preoperative Magnetic Resonance Imaging or Radiograph
}

\author{
Reinette Van Zyl, B.Sc., M.Sc., Albert-Neels Van Schoor, B.Sc., M.Sc., Ph.D., \\ Peet J. Du Toit, B.Sc., M.Sc., Ph.D., \\ Farhana E. Suleman, M.B.Ch.B., F.C.Rad. Diag, M.Med. Rad.D., \\ Mark D. Velleman, MB.Ch.B., F.C.Rad. Diag, M.Med.D, Vaida Glatt, Ph.D., \\ Kevin Tetsworth, M.D., F.R.A.C.S., and \\ Erik Hohmann, M.B.B.S., F.R.C.S., F.R.C.S. (TrEOrth), Ph.D., M.D.
}

\begin{abstract}
Purpose: To determine whether femoral epicondylar width (FECW) obtained from either magnetic resonance imaging (MRI) or plain radiographs could be used to predict anterior cruciate ligament (ACL) length. A secondary purpose was to develop a formula to use maximum FECW on either MRI or plain radiographs to estimate ACL length preoperatively. Methods: The MRIs and radiographs of 40 patients (mean age 41.0 years), with no apparent knee pathology, surgery, or trauma were included. The ACL length was measured on MRI followed by FECW on both MRI and radiograph of the same patient. This allowed the development of equations able to predict ACL length according to the FECW measured on either an MRI or radiograph. Results: The mean ACL length was $40.6 \pm 3.6 \mathrm{~mm}$. FECW measured on both MRIs and radiographs was sufficient to predict ACL length. Pearson's correlations revealed a high positive relationship between ACL length and FECW on MRI $(\mathrm{r}=0.89, P<.0001)$ and ACL length and FECW on radiograph $(\mathrm{r}=0.83, P<.0001)$. The coefficient of determination $\left(R^{2}\right)$ was calculated to be MRI: $R^{2}=0.78$ and radiograph: $R^{2}=0.68$ and confirmed that FECW measured on both MRI and radiograph were sufficient to predict ACL length. Based on these models, ACL length can be predicted by FECW using the following formulas: MRI: ACL length $=0.47$ (FECW) +1.93 and radiograph: ACL length $=0.31($ FECW $)+11.33$. Conclusions: This study demonstrated that FECW measured on either MRI or anteroposterior radiograph could reliably estimate ACL length on a sagittal MRI. There was a high positive relationship between ACL length and FECW on both MRI and radiographs, although MRIs do predict ACL length more reliably. Clinical Relevance: Preoperative ACL length assessment, using FECW on MRI or radiograph, is useful in graft selection and in preventing inadequate graft harvesting for ACL reconstruction, especially if an individualized anatomical approach is pursued.
\end{abstract}

\footnotetext{
From the Department of Anatomy, Faculty of Health Sciences (R.V.Z., A.N.V.S.), Department of Physiology, Faculty of Health Sciences, Associate of the Institute for Food, Nutrition and Well-being, Associate of the Institute for Cellular and Molecular Medicine, Associate of Sport, Exercise Medicine and Lifestyle Institute (SEMLI) (P.J.D.T.), Department of Radiology, Steve Biko Academic Hospital (F.E.S., M.D.V.), and School of Medicine (E.H.), University of Pretoria, Pretoria, South Africa; Department of Orthopaedic Surgery, University of Texas Health Science Center, San Antonio, Texas, U.S.A. (V.G.); Department of Orthopaedic Surgery, Royal Brisbane Hospital, Herston (K.T.), Department of Surgery, School of Medicine, University of Queensland, Queensland (K.T.), and Orthopaedic Research Institute of Australia, Sydney (K.T.), Australia; and Valiant Clinic/Houston Methodist Group, Dubai, United Arab Emirates (E.H.).

The authors report the following potential conflicts of interest or sources of funding: E.H., salary by the Arthroscopy Association of North America;
}

Associate Editor, Journal of Arthroscopy. Full ICMJE author disclosure forms are available for this article online, as supplementary material. Received May 28, 2019; accepted October 23, 2019.

Address correspondence to Erik Hohmann, School of Medicine, Faculty of Health Sciences, University of Pretoria, Cnr Bophelo and Dr Savage Rd., Gezina, Pretoria,0001, South Africa.E-mail:ehohmann@houstonmethodist. org

(C) 2019 THE AUTHORS. Published by Elsevier Inc. on behalf of the Arthroscopy Association of North America. This is an open access article under the CC BY-NC-ND license (http://creativecommons.org/licenses/by-nc-nd/4.0/). 2666-061X/19689

https://doi.org/10.1016/j.asmr.2019.10.005 
$\mathbf{O}$ ptimal graft choice for anterior cruciate ligament (ACL) reconstruction is still a topic of debate, but both bone-patellar tendon-bone (BPTB) and hamstring tendon (HS) autografts provide comparable and good long-term subjective and objective outcomes with low recurrence rates and a high incidence of return to sport. ${ }^{1,2}$ However, there is evidence that anterior knee pain and degenerative changes are more prevalent in BPTB grafts, ${ }^{3,4}$ whereas HS grafts may result in increased knee laxity. ${ }^{3}$ One potential issue with arthroscopic-assisted ACL reconstruction specific to BPTB grafts is the possibility of graft-tunnel mismatch. ${ }^{5-8}$ This often occurs when the length of the BPTB graft exceeds the sum of the length of the femoral and tibial tunnels and the intra-articular length between the tunnels, resulting in an extruding tibial bone plug. ${ }^{7,9,10}$ The incidence of graft-tunnel mismatch has been reported to be $26 \%^{7}$ and $13 \%$ (20\% for allografts and $10 \%$ of autografts). ${ }^{8}$

Recently, anatomic reconstruction techniques used an anteromedial portal to establish the femoral tunnel, ${ }^{11-13}$ which resulted in shorter femoral tunnels and shorter intra-articular distances. ${ }^{12,13}$ Another potential factor is the average length of the ACL, which has been recorded to be approximately 32 to $38 \mathrm{~mm} .{ }^{14-18}$ However, patellar tendon (PT) length is variable and often exceeds $50 \mathrm{~mm} .^{9,10,15,17,19}$ Patient sex and height also have been identified as risk factors for mismatch to occur. ${ }^{5,9,10}$ Nevertheless, patient height has been used as a predictor of desired graft length for BPTB allografts, ${ }^{9}$ even though some studies found a weak correlation between PT length and patient height. ${ }^{5,19}$ Meijer et al. $^{20}$ suggested that the length of the PT does not follow the expected human anthropometric trends where a tall individual will have a longer tendon. This raises the question whether a different measurement that can easily be taken could assist in predicting graft length to restore the ACL, for instance, the maximum width of the femoral epicondyles. The intraarticular length of the ACL is one of the important variables and a significant factor when selecting an appropriate graft, especially if an individualized anatomical reconstruction is pursued. ${ }^{21,22}$

High-resolution magnetic resonance imaging (MRI) is the gold standard for evaluation of ligamentous injuries, and ACL visualization is enhanced with oblique sagittal and coronal sections along the ACL's alignment. ${ }^{21}$ However, the oblique course of the ACL can make accurate measurement of the entire length of the ACL difficult, especially on a standard sagittal MRI, as the imaged region often does not cover the entire length of the ligament. An equation to assist in estimating ACL length could thus be helpful if a specialized sagittal section is unavailable. MRI is a routine part of preoperative evaluation for ACL reconstruction and should be readily available to surgeons. ${ }^{23}$ However, the reality is that the cost of MRI, the waiting periods, and the availability thereof in certain countries can be challenging. It would therefore be useful to have an alternative means by which the length of the ACL can be determined, should an MRI not be available. Simple radiographs are generally available, and Van Eck et al. ${ }^{22}$ suggested that radiographs should always be obtained to evaluate the bony morphology and the presence of any pathology. It could therefore be a reasonable alternative if radiographic variables highly correlate with ACL length. Van Zyl et al. ${ }^{15}$ demonstrated in a cadaver study that maximum femoral epicondylar width (FECW) was a more reliable predictor of ACL length than other morphologic variables such as height, and they also developed an equation to predict ACL length by measuring FECW.

The purpose of this study was therefore to determine whether FECW obtained from either MRIs or plain radiographs could be used to predict ACL length. A secondary purpose was to develop a formula to use maximum FECW on either MRI or plain radiographs to estimate ACL length preoperatively. The authors hypothesized that FECW measured on both MRI and plain radiographs would predict the ACL length as measured on a sagittal MRI accurately.

\section{Methods}

\section{Study Design}

The database of a private radiology center was searched for all patients undergoing MRI of the knee between April and July 2013. After all candidates were identified, the images were reviewed together by 2 senior radiologists with more than 20 years' experience in general radiology and 10 years' experience in musculoskeletal radiology, respectively. All patients who fulfilled the inclusion criteria were accepted into the study. The inclusion criteria were patients who presented to the hospital with nonspecific knee pain and underwent both MRI and radiographic imaging of the knee within a 3-month period; aged between 18 and 70 years old; and no readily apparent pathology, trauma, or fractures of the knee joint. The exclusion criteria were scans in which the ACL was not clearly visible, radiologic evidence of collateral or cruciate ligament injuries; previous, surgery such as meniscal resection/repair or ACL/ posterior cruciate ligament reconstruction; previous trauma, such as meniscal tears, patella fractures, tibial plateau fractures, or femoral condyle fractures; and grade 2-4 Kellgren-Lawrence classification osteoarthritis. Ethical clearance was obtained from the Research Ethics Committee, Faculty of Health Sciences, University of Pretoria, South Africa (ethics reference number 151/2013). Permission to conduct the research 
and examine patient records retrospectively also was obtained from the appropriate hospital authority.

\section{Imaging Technique}

A Philips 3T Ingenia (Philips Healthcare, Franklin, TN) MRI scanner was used for image acquisition. A dedicated radiofrequency knee coil and a slice thickness of $3 \mathrm{~mm}$ were used for all images. A minimum of 25 to 30 slices was obtained for all planes, and the following sequences were used: T2, proton density (PD), and PD with fat suppression. The MRI included sagittal, axial, and coronal images, as the ACL is imaged optimally by using multiple planes to visualize the entire length of the ACL. ${ }^{24}$ All images were taken by a single practice that uses a standardized imaging technique. All images were obtained with the patient in the supine position with the knee flexed to $10^{\circ}$, as the ACL courses along an inclined sagittal plane, and the inferior pole of the patella was positioned in the center of the knee coil. ${ }^{16}$ An anteroposterior (AP) radiograph of the knee was obtained with the patient supine having the leg extended, and both the knee and ankle in contact with the table. The patella was facing forward and the centering point was $1.5 \mathrm{~cm}$ distal to the apex of the patella, collimated to include medial and lateral skin margins, the distal femur, and the proximal tibia/fibula. All radiographs were taken according to internationally accepted protocols of radiographic techniques for knees with a standardized position and a source-to-image distance of $115 \mathrm{~cm}$ for all digital radiography. ${ }^{25}$

\section{Morphometric Variables}

Morphometric variables that were studied included ACL length on MRI and maximum FECW on both MRI and AP knee radiographs. ACL length was measured on the sagittal MRI section, using the PD-weighted sagittal image, oriented parallel to the longitudinal axis of the ACL to ensure proper visualization of the entire length of the ACL. In accordance with the study conducted by Araujo et al., ${ }^{24}$ the length of the ACL was measured at the level where both attachment sites of the ACL were visible on the selected MRI. The measurement was taken from the midpoint of the tibial insertion site to the midpoint of the femoral insertion site of the ACL (Fig 1). For FECW on MRI the PD-weighted coronal image was identified where both medial and lateral meniscal bodies and the posterior cruciate ligament femoral stump was visible, and the medial and lateral epicondyle at the widest point were connected with a line/distance tool (Fig 2). Similarly, the FECW on the AP knee radiograph was measured by connecting the widest points between the medial and lateral femoral epicondyles (Fig 3). The measurements were taken by the senior radiologist with 10 years of experience in musculoskeletal radiology and the primary author of this study. The second author reviewed all

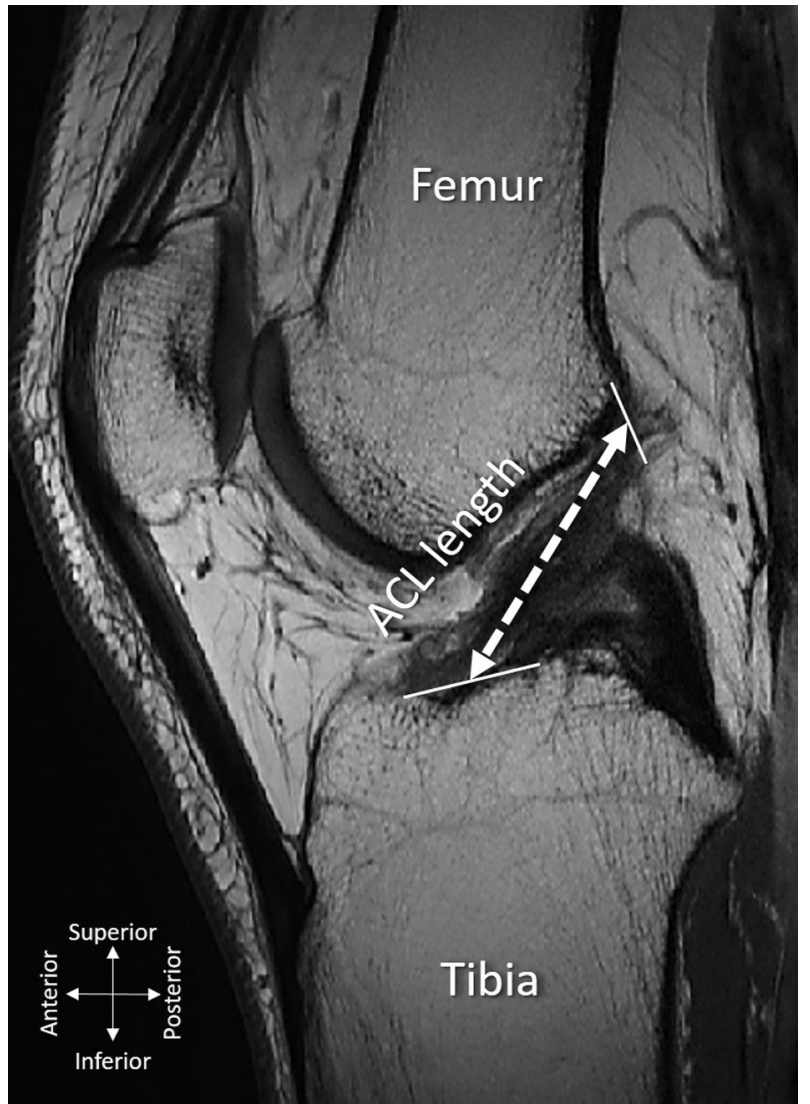

Fig 1. Sagittal magnetic resonance imaging section of the left knee that is oriented parallel to the ACL to allow proper visualization of the ACL and its attachment sites. The solid lines indicate the tibial and femoral insertion sites of the ACL respectively. ACL length is measured by connecting the midpoint of the tibial insertion site to the midpoint of the femoral insertion site of the ACL (dotted line). (ACL, anterior cruciate ligament.)

measurements for accuracy and if any major differences were present between the 2 data sets, a third measurement was taken to use the average of the measurements for the final data set. All measures were performed using the on-screen standard DICOM analysis program (GEARView Basic 2.1; PACSGEAR, Pleasanton, CA), calibrated for each image.

\section{Statistical Analysis}

Descriptive statistics were used for ACL length, the mean, standard deviation, minimum, and maximum values were determined. Boxplots were used to confirm that the collected data for each variable was symmetrically distributed. A paired $t$ test was performed to test for differences between the FECW values measured on the MRI compared with those measured on the plain radiographs.

Pearson correlation coefficients were calculated to test for pairwise associations between the ACL length and FECW on MRI and radiographs to evaluate 


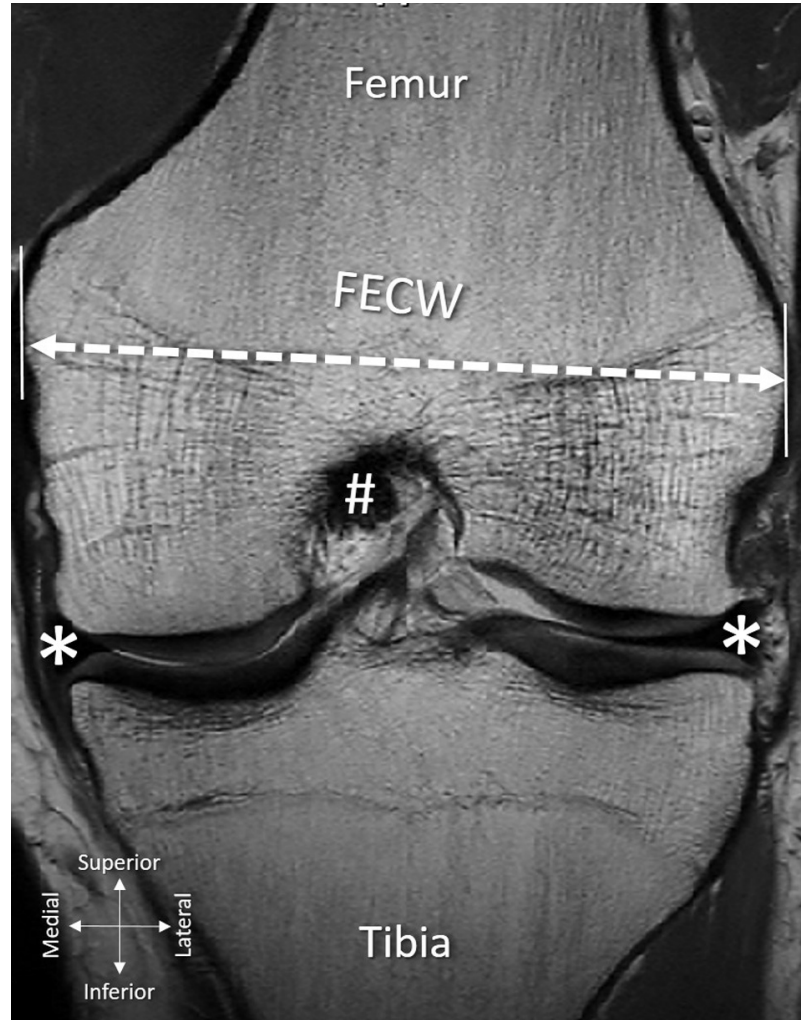

Fig 2. Coronal magnetic resonance imaging section of the left knee at the level where the meniscal bodies $\left({ }^{*}\right)$ and posterior cruciate ligament (PCL) (\#) are visible. The solid lines indicate the broadest points of the femoral epicondyles. Maximum FECW is measured by connecting the most projecting points of the medial and lateral epicondyles of the femur (dotted line). (FECW, femoral epicondylar width.)

relationships between these variables. The interpretation of the Pearson correlation coefficients by Allan ${ }^{26}$ was applied in this study: a correlation of 0.75 to 0.99 is considered high, 0.50 to 0.74 moderate, and 0.25 to 0.49 low. The coefficient of determination $\left(\mathrm{R}^{2}\right)$ was established to measure how closely the regression line approximated the real data points and if the relationship was statistically significant or not. Finally, 2 linear regression models were developed to predict ACL length according to the FECW measured on either an MRI or radiograph using SAS 9.3 for Windows (SAS Institute Inc., Cary, NC).

Intra- and interobserver reliabilities for both MRI assessment and AP knee radiographs were investigated in all 40 knees. Two investigators (R.V.Z., F.E.S.) used the described measurement methods, and intra- and interobserver correlation coefficients (intraclass correlation coefficients) were calculated. The interpretation of the intraclass correlation coefficient values by Landis and $\mathrm{Koch}^{27}$ were used in this study: below 0.0 poor, 0.00 to 0.20 slight; 0.21 to 0.40 fair; 0.41 to 0.60 moderate; 0.61 to 0.80 substantial; and 0.81 to 1.00 almost perfect.

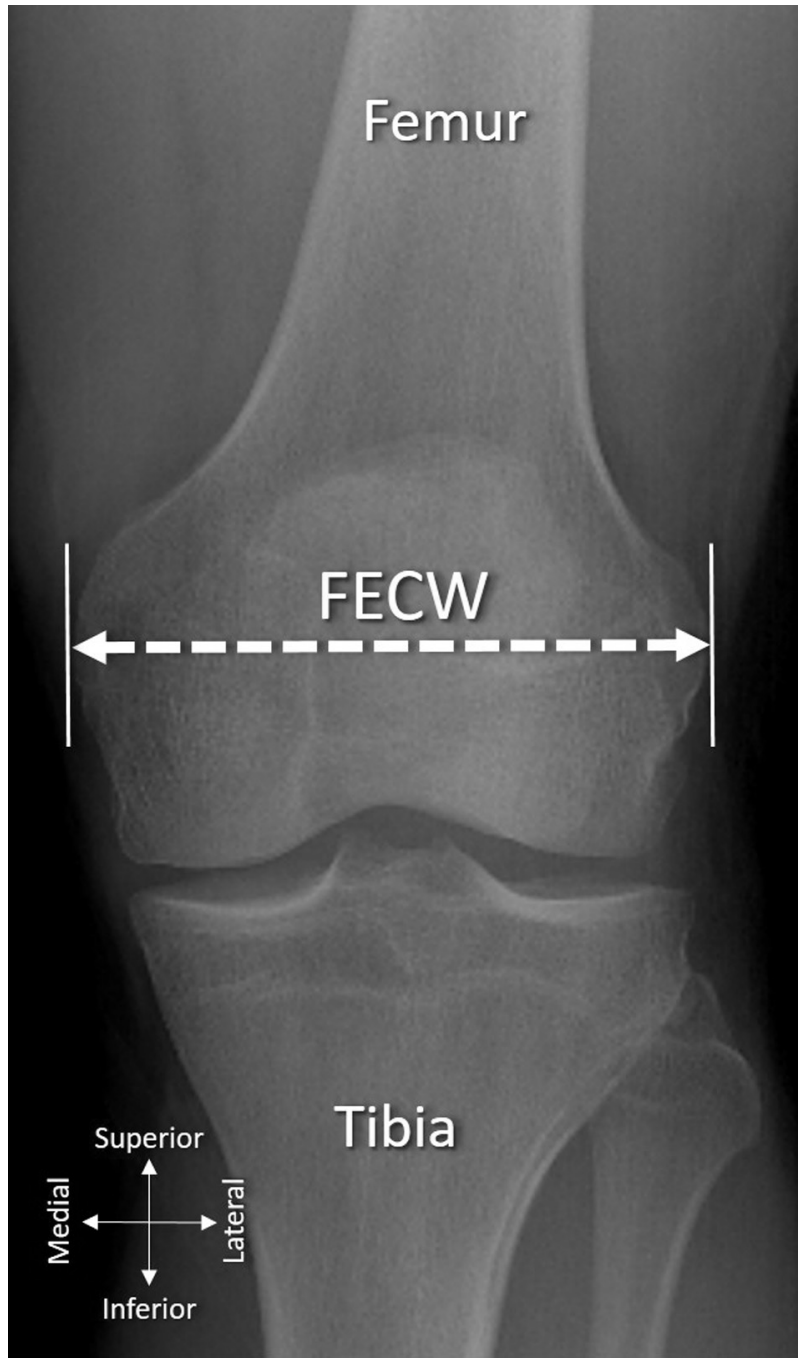

Fig 3. Anteroposterior radiograph of the left knee. The solid lines indicate the broadest points of the femoral epicondyles. Maximum FECW is measured by connecting the widest points of the medial and lateral epicondyles of the femur (dotted line). (FECW, femoral epicondylar width.)

\section{Results}

In total, 40 patients (23 male and 17 female) with a mean age of $41.0 \pm 9.9$ years (range $18-67$ years) were included in this study. The mean, standard deviation, and range of the lengths of the ACL, FECW on the MRIs, and FECW on the AP knee radiographs are demonstrated in Table 1. Pearson's moment correlations revealed a high positive relationship between ACL length and FECW on MRI $(\mathrm{r}=0.89, P<.0001)$ and ACL length and FECW on radiograph $(\mathrm{r}=0.83, P<$ .0001 ) according to the standards of Allan (Table 2). ${ }^{26}$

Boxplots confirmed symmetrical distribution of the data. The coefficient of determination $\left(R^{2}\right)$ was determined to be MRI: $\mathrm{R}^{2}=0.78$ and radiograph: $\mathrm{R}^{2}=0.68$ (Table 2). This indicated that $78 \%$ and $68 \%$ of the variability of the length of the ACL could be explained by changes in the FECW measurement on MRI or on 
Table 1. Descriptive Statistics of the MRI and Radiograph Length Measurements

\begin{tabular}{lccc}
\hline & & FECW on & $\begin{array}{c}\text { FECW on } \\
\text { Radiograph, } \mathrm{mm}\end{array}$ \\
\hline Mean & ACL Length, $\mathrm{mm}$ & MRI, mm & 94.8 \\
SD & 40.6 & 82.0 & 9.4 \\
Min. & 3.6 & 6.7 & 74.6 \\
Max. & 32.5 & 70.6 & 114.8 \\
\hline
\end{tabular}

ACL, anterior cruciate ligament; FECW, femoral epicondylar width; MRI, magnetic resonance imaging; SD, standard deviation.

radiograph, respectively. The high coefficient of determination therefore confirmed that there is a strong positive correlation between ACL length and the FECW measured on both MRI and radiograph. On the basis of these results, 2 linear regression models were derived that could possibly be used to predict ACL length according to the FECW measured on either an MRI or radiograph with the ACL length as the dependent variable (Table 3$)$.

Interobserver and intraobserver reliability for the measurements of the length of the ACL and for both the FECW on MRI and FECW on radiographs were calculated (Table 4). The intraclass correlation coefficients were "almost perfect" when interpreted according to the standards of Landis and Koch. ${ }^{27}$

\section{Discussion}

The most important finding of this study was that both FECW on MRI and AP knee radiographs could reliably predict ACL length as measured on an oblique sagittal MRI. This confirmed our hypothesis. The results also allowed for the development of 2 linear regression equations to determine the longitudinal length of the ACL before surgery by measuring FECW and applying the following formulas: MRI: ACL length $=0.47($ FECW $)+1.93$ and radiograph: ACL length $=0.31($ FECW $)+11.33$. The goal of preoperative knowledge of ACL length is to assist in graft selection for anatomical, individualized ACL reconstructive surgery.

It has been suggested that detailed preoperative planning helps to determine the most appropriate graft

Table 2. Correlation Matrix to Test for Pairwise Associations Between ACL Length and the 2 Independent Variables

\begin{tabular}{ll}
\hline & ACL Length \\
\hline FECW on MRI & $P<.0001^{*}$ \\
& $\mathrm{r}=0.89$ \\
FECW on radiograph & $\mathrm{R} 2=0.78$ \\
& $P<.0001^{*}$ \\
& $\mathrm{r}=0.83$ \\
& $\mathrm{R}^{2}=0.68$ \\
\hline
\end{tabular}

ACL, anterior cruciate ligament; FECW, femoral epicondylar width; MRI, magnetic resonance imaging.

*Statistically significant correlation.
Table 3. Equations to Predict ACL Length by Means of the Maximum FECW Measured on Either MRI or Radiograph

\begin{tabular}{lc}
\hline \multicolumn{1}{c}{ MRI } & \multicolumn{1}{c}{ Radiograph } \\
\hline ACL length $=0.47($ FECW $)+1.93$ & ACL length $=0.31$ \\
$($ FECW $)+11.33$ \\
\hline ACL, anterior cruciate ligament; FECW, femoral epicondylar width; \\
MRI, magnetic resonance imaging
\end{tabular}

for ACL reconstruction and the ability to preoperatively template the appropriate graft size will minimize graft-tunnel mismatch. ${ }^{20,21,28,29}$ Factors that influence graft choice include bone morphology, size of the intercondylar notch, and the tibial and femoral footprints of the ACL. ${ }^{28}$ Two common grafts that frequently are used are HS and BPTB grafts, and excellent results can be obtained with both if surgical techniques are consistently applied. ${ }^{1,3,4}$ However, BPTB grafts are often too long and could extrude from the tibial bone plug, resulting in graft mismatch. ${ }^{7,9,10}$ In contrast, HS grafts could potentially have small diameters, ${ }^{30,31}$ and a diameter of $8 \mathrm{~mm}$ or less results in greater failure rates. $^{31,32}$ Quadriceps tendon ${ }^{33}$ and patellar bone-tendon grafts ${ }^{6}$ also have been described as possible options. Gilmer suggested that accurate preoperative assessment is more important than ever to prevent inadequate graft size before harvest. ${ }^{23}$ If accurate information about intra-articular ACL length is known preoperatively, it could assist in making informed decisions about graft selection of adequate length for the specific patient. The mean ACL length was measured to be $40.6 \mathrm{~mm}$ on the oblique sagittal MRIs. The values obtained in this study were greater than those previously reported in the literature, where the mean lengths were documented as 32 to 38 mm. ${ }^{14-18}$ It should be noted that Van Zyl et al. ${ }^{15}$ used cadaveric knees and measured ACL length along its anterior border with the knee flexed to $90^{\circ}$, whereas this study used sagittal MRIs with the knee flexed to $10^{\circ}$ to visualize the ACL along its longitudinal axis. It is known that the position of the knee influences the length of the $\mathrm{ACL}^{14,34}$; it is longest at maximum extension and shortest at 136 and $125^{\circ}$ flexion for the anteromedial and posterolateral bundles respectively. ${ }^{35}$

Table 4. ICCs for Both the MRI Assessments and the AP Radiographs

\begin{tabular}{|c|c|c|c|c|}
\hline & \multicolumn{2}{|c|}{ Interobserver Reliability } & \multicolumn{2}{|c|}{ Intraobserver Reliability } \\
\hline & Mean ICC & $95 \% \mathrm{CI}$ & Mean ICC & $95 \% \mathrm{CI}$ \\
\hline$\overline{\text { ACL length }}$ & 0.92 & $0.86-0.96$ & 0.92 & $0.85-0.95$ \\
\hline FECW on MRI & 0.98 & $0.96-0.99$ & 0.99 & $0.98-0.99$ \\
\hline $\begin{array}{l}\text { FECW on } \\
\text { radiograph }\end{array}$ & 0.98 & $0.96-0.99$ & 0.98 & $0.96-0.99$ \\
\hline
\end{tabular}


This could explain this discrepancy. Wang et al. ${ }^{16}$ used the same knee positioning during their sagittal MRI scan than this study, but they explained their ACL length measurement as being from the highest point of the intercondylar fossa of the femur to the front facies ossea of the intercondylar eminence of the tibia, which is more toward the anterior border of the ACL. This differs from our measurement between the midpoints of the ACL attachments sites and may account for the difference in mean length.

Furthermore, measurement of ACL length on MRI is complicated, as the ACL is not a perfectly sagittal structure and may be miscalculated when measured on a single standard sagittal section. ${ }^{10}$ Markatos et al. ${ }^{18}$ stated that the ACL is best visualized on 2 or 3 sagittal MRI sections, and Högerle et al. ${ }^{17}$ illustrated the full length of the ACL by reconstructing 3 MRI sections of the knee and stated the technique provided complete information of volume. However, the latter study was completed more than 20 years ago. The current study used sagittal sections that were aligned with the longitudinal axis of the ACL, indicating both attachment sites, to account for the oblique course of the ligament in question. These aforementioned findings confirm the variability of ACL measurement techniques, and therefore surgeons should make sure of knee position, MRI scanning technique, especially related to the sagittal section, and the exact end points when measuring ACL length.

However, ACL variability between individuals is still an important point to consider when performing anatomical reconstruction, ${ }^{14}$ as anatomic reconstruction entails restoring the ACL to its original dimensions. ${ }^{22}$ This is relevant to the insertion sites, ${ }^{21}$ but re-establishing the native length could also be important, for instance when considering the native tension patterns of the ligament. Preoperative ACL length estimation could be an asset to surgeons, as it will allow surgeons greater confidence in evaluating and counseling patients regarding the selection of an appropriate graft of sufficient dimensions for their anatomic reconstruction, not only to re-establish the thickness, insertion footprints, and inclination angle, but also the original length of the ACL.

Several previous studies have expressed the need for using independent factors, such as height, weight, and sex of a population to determine intra-articular ACL length. ${ }^{5,9,15}$ Brown et al. ${ }^{9}$ demonstrated a strong correlation between patient height and the prediction of the required length of the tendinous portion of the BPTB graft necessary for ACL reconstruction. In contrast, Denti et al. ${ }^{5}$ were unable to confirm the relationship between ACL length and patient height, as well as failed to observe a relationship between PT length and patient height. Similarly, Zakko et al. ${ }^{29}$ could only demonstrate weak correlations between anthropometric data (height, weight, body mass index, age, and sex) and the size of hamstring, quadriceps, and PTs. This was further confirmed by the moderate positive and weak correlations observed between patient height and PT length for any given height by Goldstein et al. ${ }^{10}$ and Navali and Jafarabadi, ${ }^{19}$ respectively. It is suggested that PT length does not follow the expected human anthropometric trends ${ }^{19,20}$ and that preoperative MRI measurements should rather be considered. ${ }^{19}$ Therefore, using anthropometric variables may not be practical. In addition, height and weight are often self-reported by the patient, and this reduces the accuracy and reliability of these variables. ${ }^{29}$ However, plain radiographs and MRI are commonly used during routine preoperative assessment, and maximum FECW can be easily measured on these images. Therefore, it may be a very helpful tool in determining intra-articular ACL length.

The mean maximum FECW measured on a coronal MRI was $82.0 \mathrm{~mm}$ and on AP radiograph $94.8 \mathrm{~mm}$. The FECW on radiographs were thus greater than the comparable MRI measurement. This could be due to the magnification difference between these 2 imaging modes, as the MRI had no magnification factor, whereas the radiographs had a source-to-image distance of $115 \mathrm{~cm}$. Nevertheless, the Pearson correlation coefficient revealed that the variables (FECW on MRI and FECW on plain radiographs) demonstrated significant and high positive linear relationships with ACL length. The data have established that the FECW measured on either MRI or plain radiographs was a satisfactory predictor of longitudinal ACL length as measured on a sagittal MRI and had high $r$ values above 0.8 . Van Zyl et al. ${ }^{15}$ previously measured the relationship between FECW and ACL length in a cadaver study, but in their study the r value was only 0.36 ; this was probably due to their method of ACL measurement along the anterior border in a knee flexed to $90^{\circ}$, resulting in shorter ACL lengths. The best $r$ value in this study, of FECW measured on MRI, was further confirmed by the coefficient of determination $\mathrm{R}^{2}$ value of 0.78 . This implied that the equation used could account for $78 \%$ of the variations found in ACL length. This proves that determining the FECW on a coronal MRI is more accurate, and therefore, the best predictor for estimating ACL length. However, the FECW measured on a radiograph had an $\mathrm{R}^{2}$ value of 0.68 , which was slightly lower but can still be considered a very good predictor of ACL length.

On the basis of these results, we have used 2 linear regression models to predict ACL length, as measured on an oblique sagittal MRI, with regards to FECW measured on either an MRI or AP radiograph. The reason for developing 2 equations could be explained by the difference between the FECW measurements on 
MRI and radiograph, but even though there was a known magnification difference, the equations accounted for this factor. Therefore, if an MRI is used to obtain maximum FECW, the MRI equation should be used, and if a standard radiograph is used, the radiographic counterpart. The equation developed by Van Zyl et al. ${ }^{15}$ differed from those in this study, but this could be explained by their method of ACL measurement along the anterior border in a knee flexed to $90^{\circ}$. Nevertheless, both of the equations developed in this study allow reliable prediction of longitudinal ACL length and can be used to plan graft length requirements preoperatively by allowing the surgeon to estimate the intra-articular length that needs to be restored. This information could assist in selecting the appropriate graft for the specific patient, especially to prevent an excessively long graft that could protrude from the tibial tunnel. It would also provide a parameter when ordering allografts, as Goldstein et al. ${ }^{10}$ commented that the risk of graft-tunnel mismatch might be decreased if an appropriate length graft is requested. For example, if a patient has a short ACL, a BPTB graft might be too long and other alternatives, like a quadriceps tendon or HS graft, could be considered.

Multiple studies have documented methods to determine the size of graft types before surgery. ${ }^{6,16,24,29,36}$ This information should be used to determine the available tendon sizes to establish whether harvest of that tendon type is feasible before surgery. ${ }^{29}$ Combined with knowledge of the length of the native ACL that needs to be restored, the adequate graft could be estimated. Brown et al. ${ }^{9}$ suggested the addition of $10 \mathrm{~mm}$ to the predicted intra-articular ACL length to allow some flexibility for fixation and an additional $50 \mathrm{~mm}, 25 \mathrm{~mm}$ for each bone plug. However, Goldstein et al. ${ }^{10}$ were concerned that this method would result in ordering grafts that are too long, again resulting in increased risk of mismatch. Meijer et al. ${ }^{20}$ added $20 \mathrm{~mm}$ to achieve the overall tendinous length when ordering an allograft $(10 \mathrm{~mm}$ at each end to make the turns at the tibial and femoral apertures). This information could then be used to predict the required graft length (combined intraarticular, fixation, and bone tunnel lengths) and thus the graft type that would be sufficient for an individualized and anatomical ACL reconstruction with a lowered risk of graft mismatch. Because most surgeons obtain a radiograph and/or MRI scan before ACL reconstruction, this method of ACL length prediction may be added to routine preoperative planning with a small addition in protocol.

The length of the ACL is difficult to measure due to its oblique course ${ }^{10}$ and an oblique sagittal MRI that runs parallel to the ACL is needed to do so. ${ }^{24} \mathrm{~A}$ formula to estimate intra-articular ACL length would therefore be helpful if such a specialized MRI section is not available. Using a simple anatomic measure, such as FECW on either MRIs or routine AP radiographs, enables reliable and simple prediction of ACL length. This may allow the surgeon to predict the total required graft length and select the appropriate graft for reconstruction, especially if an individualized, anatomical approach is pursued.

\section{Limitations}

This study has certain limitations. Cross-validation of the equations derived was not performed, and these would benefit from further evaluation to confirm the accuracy of the prediction model. Rather than asymptomatic volunteers, the MRIs of patients with knee symptoms, although seemingly normal ACLs and no apparent knee pathology, were used. The patients had undiagnosed knee pain and this may have biased results in ways that would be difficult to determine. Although all scans were thoroughly examined for pathology of the intra- and extraarticular ligaments, the possibility of occult injuries cannot be excluded. The inclusion and exclusion criteria were strictly defined, but they cannot entirely exclude the possibility that the ACL was compromised. Further, this study did not distinguish between male and female patients, and sex has been described as a determinant of ACL size. ${ }^{14}$ Studies have found significant differences of ACL lengths between the sexes, where male patients presented with longer ACLs. ${ }^{16,37}$

\section{Conclusions}

This study demonstrated that FECW measured on either MRI or AP radiograph could reliably estimate ACL length on a sagittal MRI. There was a high positive relationship between ACL length and FECW on both MRI and radiographs, although MRIs do predict ACL length more reliably.

\section{References}

1. Webster KE, Feller JA, Hartnett N, Leigh WB, Richmond AK. Comparison of patellar tendon and hamstring tendon anterior cruciate ligament reconstruction: A 15-year follow-up of a randomized controlled trial. Am J Sports Med 2016;44:83-90.

2. Gabler CM, Jacobs CA, Howard JS, Mattacola CG, Johnson DL. Comparison of graft failure rate between autografts placed via an anatomic anterior cruciate ligament reconstruction technique: A systematic review, meta-analysis, and meta-regression. Am J Sports Med 2016;44:1069-1079.

3. Ciccotti MC, Secrist E, Tjoumakaris F, Ciccotti MG, Freedman KB. Anatomic anterior cruciate ligament reconstruction via independent tunnel drilling: A systematic review of randomized controlled trials comparing 
patellar tendon and hamstring autografts. Arthroscopy 2017;33:1062-1071.

4. Poehling-Monaghan KL, Salem H, Ross KE, et al. Longterm outcomes in anterior cruciate ligament reconstruction: A systematic review of patellar tendon versus hamstring autografts. Orthop J Sports Med 2017;5: 2325967117709735.

5. Denti M, Bigoni M, Randelli P, et al. Graft-tunnel mismatch in endoscopic anterior cruciate ligament reconstruction. Intraoperative and cadaver measurement of the intra-articular graft length and the length of the patellar tendon. Knee Surg Sports Traumatol Arthrosc 1998;6:165-168.

6. Kim S-J, Yoon J-Y, Kim S-M, Ha S, Kim S-H, Cho I. Comparative study on the postoperative outcomes of anterior cruciate ligament reconstruction using patellar bone-tendon autografts and bone-patellar tendon-bone autografts. Arthroscopy 2016;32:1072-1079.

7. Shaffer B, Gow W, Tibone JE. Graft-tunnel mismatch in endoscopic anterior cruciate ligament reconstruction: A new technique of intraarticular measurement and modified graft harvesting. Arthroscopy 1993;9:633-646.

8. Verma NN, Dennis MG, Carreira DS, Bojchuk J, Hayden JK, Bach BR. Preliminary clinical results of two techniques for addressing graft tunnel mismatch in endoscopic anterior cruciate ligament reconstruction. J Knee Surg 2005;18:183-191.

9. Brown JA, Brophy RH, Franco J, et al. Avoiding allograft length mismatch during anterior cruciate ligament reconstruction: Patient height as an indicator of appropriate graft length. Am J Sports Med 2007;35:986-989.

10. Goldstein JL, Verma N, McNickle AG, Zelazny A, Ghodadra N, Bach BR. Avoiding mismatch in allograft anterior cruciate ligament reconstruction: Correlation between patient height and patellar tendon length. Arthroscopy 2010;26:643-650.

11. Fu FH, van Eck CF, Tashman S, Irrang JJ, Moreland MS. Anatomic anterior cruciate ligament reconstruction: A changing paradigm. Knee Surg Sports Traumatol Arthrosc 2015;23:640-648.

12. Lubowitz JH, Konicek J. Anterior cruciate ligament femoral tunnel length: Cadaveric analysis comparing anteromedial portal versus outside-in technique. Arthroscopy 2010;26:1357-1362.

13. Osti M, Krawinkel A, Ostermann M, Hoffelner T, Benedetto KP. Femoral and tibial graft tunnel parameters after transtibial, anteromedial portal, and outside-in single-bundle anterior cruciate ligament reconstruction. Am J Sports Med 2015;43:2250-2258.

14. Irarrazaval S. Variations in anterior cruciate ligament anatomy. Oper Tech Orthop 2017;27:8-13.

15. Van Zyl R, Van Schoor A-N, Du Toit PJ, Louw EM. Clinical anatomy of the anterior cruciate ligament and pre-operative prediction of ligament length. SA Orthop J 2016;15:47-52.

16. Wang HP, Cui HK, Yue W, et al. Determination of patellar ligament and anterior cruciate ligament geometry using MRI. Genet Mol Res 2015;14:12352-12361.
17. Högerle S, Letsch R, Sievers KW. ACL reconstruction by patellar tendon: A comparison of length by magnetic resonance imaging. Arch Orthop Trauma Surg 1998;117: 58-61.

18. Markatos K, Kaseta MK, Lallos SN, Korres DS, Efstathopoulos N. The anatomy of the ACL and its importance in ACL reconstruction. Eur J Orthop Surg Traumatol 2013;23:747-752.

19. Navali AM, Jafarabadi MA. Is there any correlation between patient height and patellar tendon length? Arch Bone Jt Surg 2015;3:99-103.

20. Meijer K, Saper M, Joyner P, Liu W, Andrews JR, Roth C. Minimizing graft-tunnel mismatch in allograft anterior cruciate ligament reconstruction using Blumensaat's line: A cadaveric study. Arthroscopy 2018;34: 2438-2443.

21. Hofbauer M, Muller B, Murawski CD, Van Eck CF, Fu FH. The concept of individualized anatomic anterior cruciate ligament (ACL) reconstruction. Knee Surg Sports Traumatol Arthrosc 2014;22:979-986.

22. Van Eck CF, Lesniak BP, Schreiber VM, Fu FH. Anatomic single- and double-bundle anterior cruciate ligament reconstruction flowchart. Arthroscopy 2010;26:258-268.

23. Gilmer BB. Editorial commentary: Goldilocks and the three grafts: Managing tendon harvest and graft length problems in autograft anterior cruciate ligament reconstruction. Arthroscopy 2018;34:2463-2465.

24. Araujo P, van Eck CF, Torabi M, Fu FH. How to optimize the use of MRI in anatomic ACL reconstruction. Knee Surg Sports Traumatol Arthrosc 2013;21:1495-1501.

25. Bontrager KL, Lampignano JP. Bontrager's handbook of radiographic positioning and techniques. St Louis, MO: Elsevier Mosby, 2013.

26. Allan JH. Learning about statistics. New York: Macmillan, 1982.

27. Landis JR, Koch GG. The measurement of observer agreement for categorical data. Biometrics 1977;33: 159-174.

28. Iriuchishima T, Ryu K, Aizawa S, Fu FH. Proportional evaluation of anterior cruciate ligament footprint size and knee bony morphology. Knee Surg Sports Traumatol Arthrosc 2015;23:3157-3162.

29. Zakko P, van Eck CF, Guenther D, Irrgang JJ, Fu FH. Can we predict the size of frequently used autografts in ACL reconstruction? Knee Surg Sports Traumatol Arthrosc 2017;25:3704-3710.

30. Frank RM, Higgins J, Bernardoni E, et al. Anterior cruciate ligament reconstruction basics: Bone-patellar tendon-bone autograft harvest. Arthrosc Tech 2017;6: el189-el 194.

31. Ramkumar PN, Hadley MD, Jones MH, Farrow LD. Hamstring autograft in ACL reconstruction: A 13-year predictive analysis of anthropometric factors and surgeon trends relating to graft size. Orthop J Sports Med 2018;6:1-7.

32. Magnussen RA, Lawrence JTR, West RL, Toth AP, Taylor DC, Garrett WE. Graft size and patient age are predictors of early revision after anterior cruciate ligament 
reconstruction with hamstring autograft. Arthroscopy 2012;28:526-531.

33. Xerogeanes JW. Quadriceps tendon graft for anterior cruciate ligament reconstruction: The graft of the future! Arthroscopy 2019;35:696-697.

34. Iwahashi T, Shino K, Nakata K, et al. Assessment of the "functional length" of the three bundles of the anterior cruciate ligament. Knee Surg Sports Traumatol Arthrosc 2008; 16:167-174.

35. Hino K, Shiraishi Y, Nishimatsu K, et al. In vivo cruciate ligament length pattern assessment secondary to differences in femoral attachment under loading condition using image-matching techniques. J Orthop Sci 2019;24:294-300.

36. Ilahi OA, Staewen RS, Stautberg EF, Qadeer AA. Estimating lengths of semitendinosus and gracilis tendons by magnetic resonance imaging. Arthroscopy 2018;34: 2457-2462.

37. Estes K, Cheruvu B, Lawless M, Laughlin R, Goswami T. Risk assessment for anterior cruciate ligament injury. Arch Orthop Trauma Surg 2015;135: 1437-1443. 